\title{
Determining the true slip of a yield stress material with a sliding plate rheometer
}

\author{
Christian Clasen
}

Department of Chemical Engineering, University of Leuven (KU Leuven), Willem de Croylaan 46, 3001 Heverlee, Belgium

\begin{abstract}
Within this paper we demonstrate the capability of the sliding plate configuration of the flexure-based microgap rheometer (FMR) to absolutely determine slip velocities of a yield stress fluid. The sensitivity of the compound flexures of the FMR in combination with the possibility to achieve precise gap settings down to $1 \mu \mathrm{m}$ allows to accurately determining slip velocities down to $1 \mu \mathrm{m} / \mathrm{s}$. We further show how the obtained non-linear relation of the slip velocity to the constant stresses of the plane Couette flow in the sliding plate configuration of the FMR allows predicting and explaining the inhomogeneous stress distribution and partial yielding behaviour in a rotational cone-and-plate geometry.
\end{abstract}

\section{Introduction}

The slip of sheared simple and complex fluids in the vicinity of a solid wall has been the subject of intensive studies since the first introduction of this phenomenon by Navier in the early $19^{\text {th }}$ century (Navier 1823; Piau and Piau 2005). Next to the discussions of the origin of slip depending on the structure of the liquid and surface (Neto et al. 2005; Barnes 1995), and the effects of slip on the processing and applications of these fluids (Laun 2004; Kalika and Denn 1987), it is in particular the precise determination of the actual slip that has been the focus of experimental rheologists (Barnes 1995). 
The amount of slip in a sheared fluid is generally experimentally determined with a Couette or plate-and-plate (PP) geometry on a rotational rheometer using the Mooney analysis (Mooney 1931; Yoshimura and Prudhomme 1988). In case that the slip velocity $v_{s}$ of the bulk fluid against a wall can be considered to be a function of the stress $\sigma$, it is possible to determine $v_{s}$ with the Mooney analysis from the distribution of slip velocity and bulk shear rate $\dot{\gamma}_{b}$ from the nominal total shear rate $\dot{\gamma}_{n}=\dot{\gamma}_{b}+2 v_{s} / h$ with measurements at different gap distances $h$ but the same stress level $\sigma$ (Yoshimura and Prudhomme 1988). Such a change in the measurement gap distance $h$ can be achieved for a rotational rheometer with Couette type geometries of different cup or bob diameters. However, the availability such different geometries is normally limited and thus also the analysis of the slip. The use of a rotational PP geometry enables in principle an easy adjustment of the gap to different levels, however, the shear rate and thus the stress are in this type of geometry not constant but vary in radial direction. A slip analysis using a rotational PP geometry is thus inherently operating with averaged values and the error induced is in particular large for systems that show a strong dependence of the slip velocity on the applied stress.

In order to obtain an unambiguous determination of slip, there have been several attempts to directly measure slip. Approaches include direct marking and visualization of the free sample interface in rotational geometries (Princen and Kiss 1986; Kalyon et al. 1993), heterodyne dynamic light scattering (Salmon et al. 2003), NMR velocity imaging (Mair and Callaghan 1996), ultrasonic speckle or doppler velocimetry (Manneville et al. 2004; Derakhshandeh et al.) and particle imaging velocimetry (Lumma et al. 2003; Meeker et al. 2004a; Dimitriou et al. 2011; Seth et al. 2008). However, even for an exact determination of an absolute slip velocities and/or lengths, the stresses that are calculated in PP or cone-and-plate (CP) geometries from the simultaneously measured torque $T$ represent only an averaged value over the inherently inhomogeneous radial distribution of stresses for a slipping sample. These 
'apparent' stresses prohibit an exact determination of the absolute stresses that are related to the slip phenomena, allowing thus only a qualitative comparison to the theoretical predictions.

This difficulty can be overcome by creating a planar Couette flow using a sliding plate rheometer. The constant and absolutely measured stresses between the parallel plates of such a device as well as the adjustable gap allow a precise analysis of a slipping phenomenon. For higher viscosity systems in particular the sliding plate devices developed by Dealy and coworkers (Giacomin et al. 1989; Hatzikiriakos and Dealy 1991) have been used for studies of the wall slip of polymer melts and solutions. With the recently introduced Flexure-based Microgap Rheometer (FMR) (Clasen and McKinley 2004; Clasen et al. 2010; Baik et al. 2011; Clasen et al. 2006; Kojic et al. 2006; Baik et al. 2008; Erni et al. 2011) a sliding plate rheometer was developed that can also investigate lower viscosity systems, and that can in particular access gaps between the sliding plates down to $1 \mu \mathrm{m}$. This enables a precise slip analysis in particular when low slipping velocities of order $1 \mu \mathrm{m} / \mathrm{s}$ are present (Clasen et al. 2006) as they are encountered in polymer solutions or colloidal suspensions.

In this paper we will demonstrate how the micrometer gap distances in the parallel plate geometry of the FMR enable an accurate analysis of the non-linear evolution of the slip velocity with the stress. The sample selected for this study is a concentrated solution of the polysaccharide xanthan gum that exhibits a yield stress due to hydrogen bonding. Yield stress fluids as xanthan gum have been the aim of many slip studies (Bertola et al. 2003; Rofe et al. 1996), as they exhibit no or negligible flow in the bulk phase below the yield stress, allowing to focus solely on the slip phenomenon. Furthermore, in comparison to microgel pastes and concentrated emulsions that have been studied in detail as model yield stress fluids (Meeker et al. 2004a), for xanthan gum one can rule out confinement effects from microstructures of the order of the gap settings used for the slip study with the FMR (Davies and Stokes 2008; Clasen and McKinley 2004; Clasen et al. 2006). In addition to the accurate slip analysis with 
the FMR, we show that it is possible based on the FMR analysis to calculate and explain the radial distribution of stresses of the slipping yield stress fluid in a regular cone-and-plate geometry and to compare the total theoretical torque to the experimentally observed apparent stresses as function of the nominal shear rate.

\section{Experiments and Methods}

The FMR sliding plate rheometer used in this study utilizes a compound-flexure based drive/sensing system that allows to maintain the parallelism and separation of the plates even for $\mu \mathrm{m}$ gap separations over large shearing deformations. The technical details of the FMR used in this study are given elsewhere (Clasen et al. 2006), with a detailed analysis of its limitations in (Clasen et al. 2010) and (Baik et al. 2011). The square shearing surfaces used in the FMR for this study consist of optically flat glass $(\lambda / 4)$ with a surface area $A$ of $217 \mathrm{~mm}^{2}$. Each data point of the FMR measurements is obtained in a controlled rate mode and represents a steady state stress within $3 \%$ over a time period of at least $3 / \dot{\gamma}$.

Rotational rheometrical measurements were conducted with an AR-G2 (TA Instruments) rotational rheometer, using the same steady state conditions for the stress as a function of applied shear rate as for the sliding plate rheometer. The geometries used were a cone-andplate (CP) geometry with an angle $\alpha$ of $2^{\circ}$ and a radius $R$ of $2 \mathrm{~cm}$, and a plate-and-plate (PP) geometry, also with a radius $R$ of $2 \mathrm{~cm}$.

The sample investigated in this study is a $5 \mathrm{wt} \%$ aqueous solution of xanthan gum (Keltrol BT, CP Kelco) with 0.012 wt $\% \mathrm{NaN}_{3}$ added as an antibacterial agent. A non-thixotropic behaviour of the solution over the timescales applied was confirmed by measuring the sample with the cone-and-plate geometry consecutively with increasing and decreasing rates.

Measurements with the FMR as well as with the rotational rheometer were conducted at an ambient room temperature of $23^{\circ} \mathrm{C}$. 
MATLAB (Mathworks) was used to perform the numerical integration to solve eq. (2).

\section{Results and Discussion}

Fig. 1 shows the apparent stresses $\sigma=3 T /\left(2 \pi R^{3}\right)$ determined from the measured torque $T$ for a CP geometry (Macosko 1994) as a function of the nominal shear rates $\dot{\gamma}_{n, c p}=\Omega / \tan \alpha$ calculated from the applied angular velocity $\Omega$ that are obtained for the Xanthan gum solution. Figure 1 gives also the apparent stresses $\sigma=2 T /\left(\pi R^{3}\right)$ measured with the PP geometry (Macosko 1994) at different gap settings $h$ from 20 to $1000 \mu \mathrm{m}$ as a function of the nominal shear rate at the $\operatorname{rim} \dot{\gamma}_{n, p p}=\Omega R / h$. All flow curves show at a stress level of approximately $140 \mathrm{~Pa}$ a nearly rate independent plateau that can be attributed to a yielding of the material at this critical stress level $\sigma_{y}$. At higher rates, the flow curves obtained with the different geometries nearly coincide and show a shear thinning behaviour as expected for an aqueous solution of xanthan gum. For both the CP and PP geometries the yield stress plateau does not extend to small rates and instead over a range of shear rates from $10^{-2}-10^{1} \mathrm{~s}^{-1}$ an apparent shear thinning flow regime is observed that shifts to higher rates for decreasing gaps for the PP data. For the PP geometry at lower gaps, in particular $50 \mu \mathrm{m}$ and $20 \mu \mathrm{m}$, it should be noted that $h$ (and therefore the shear rate $\dot{\gamma}_{p p}=\Omega R / h$ ) is only indicative for the actual gap, since no correction of gap errors due to non-parallelism of the plates has been performed (Davies and Stokes 2008; Clasen 2012; Kramer et al. 1987). A final thing to note for the PP data at gaps larger than $250 \mu \mathrm{m}$ is a slight onset of a shoulder that can be observed on close inspection at a stress level of $\sigma \sim 70 \mathrm{~Pa}$ and that can also be seen in the $\mathrm{CP}$ data, but that is not visible for the smaller PP gaps and also not in the FMR data at even smaller gaps presented in the following. It can be speculated that the origin of this discontinuity is a supramolecular structure that can only build up in larger gaps; however, this is not of importance for the following discussion of the slip phenomenon of a yield stress fluid. 


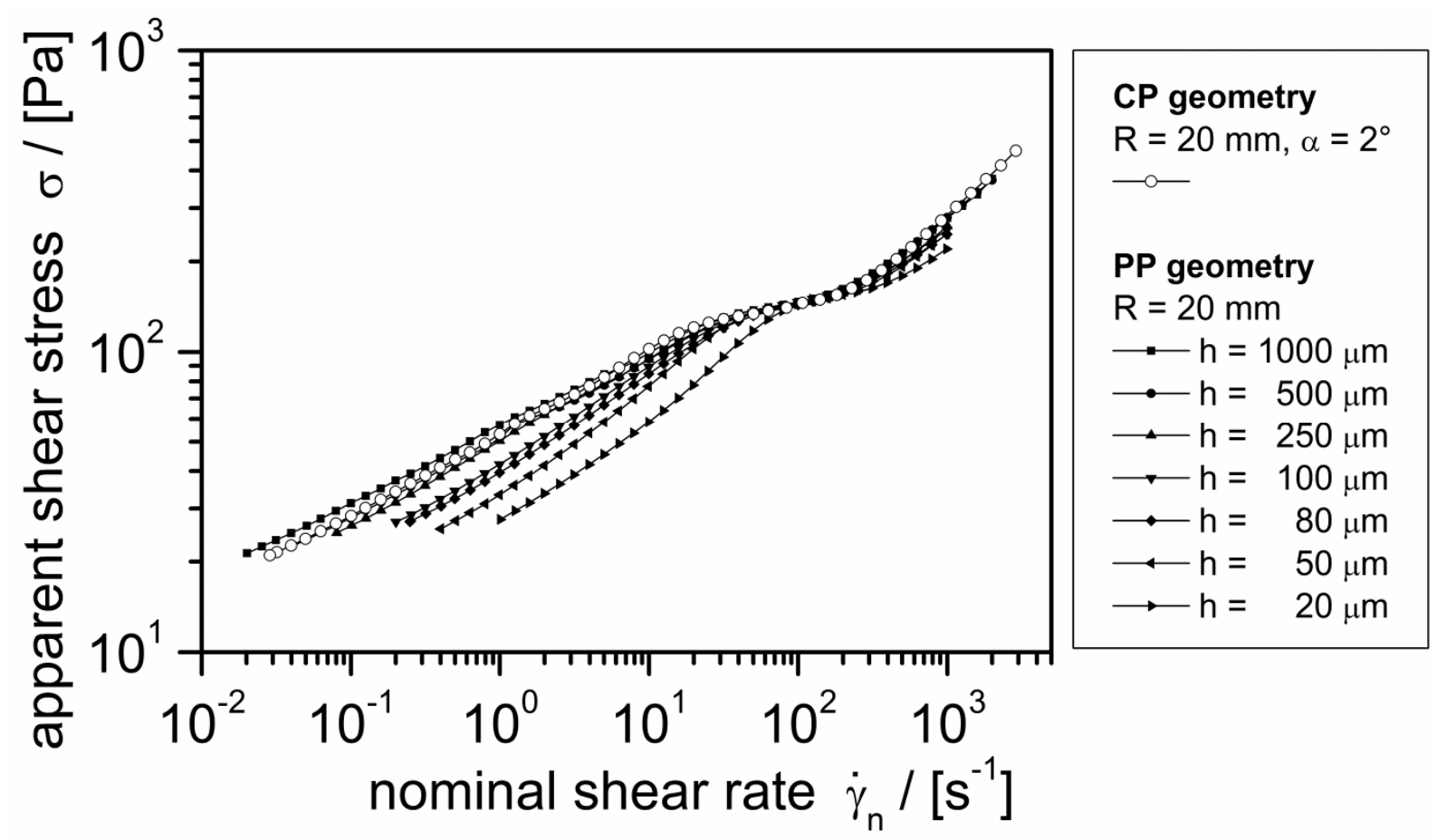

Fig. 1 Apparent shear stress of the $5 \mathrm{wt} \%$ aqueous xanthan gum solution determined with a cone-andplate (CP) geometry as well as a plat-and-plate (PP) geometry at different gaps $h$, as a function of the nominal shear rate of the respective geometry.

The relatively large nominal shear rates, as well as the dependence on the gap for the flow curves of the PP experiments, indicate that the apparent shear thinning in the low shear regime is not a creeping flow of the unyielded sample, but rather a slip phenomenon of the unyielded material against the shearing surface or within the bulk. Furthermore, the general shape of the flow curves in Fig. 1 has already been reported and possibly attributed to slip for other complex fluids with a yield stress as for example concentrated microgel (Meeker et al. 2004b, a) or fibre pulp suspensions (Derakhshandeh et al.), mayonnaise (Clasen et al. 2006) or toothpaste (Ardakani et al. 2011). This slip phenomenon is investigated in the following in detail with the sliding plate geometry and the constant small gaps of the FMR. 


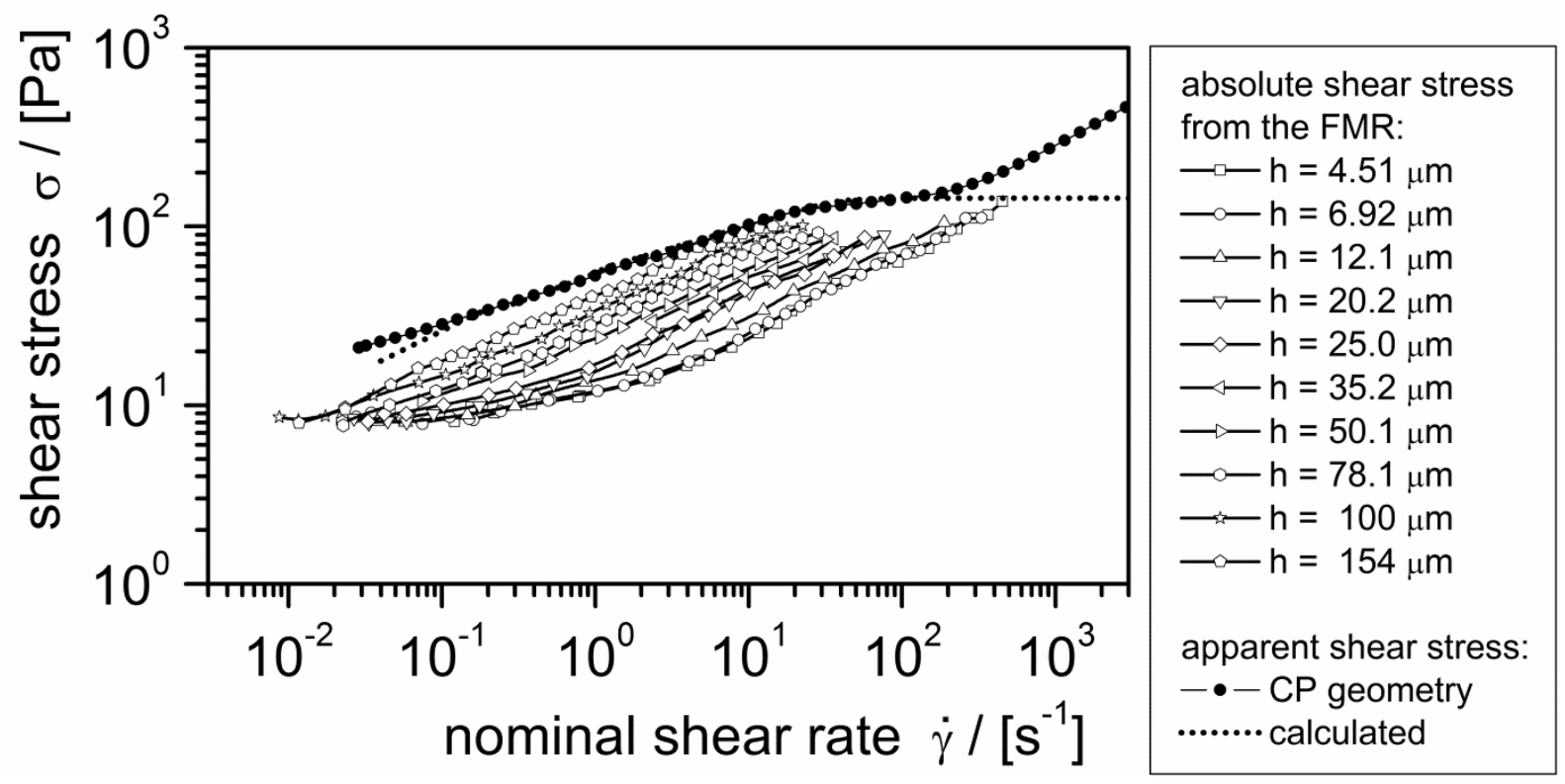

Fig. 2 Absolute shear stresses of the $5 \mathrm{wt} \%$ aqueous xanthan gum solution as a function of the nominal shear rates determined with the sliding plate geometry of the FMR (open symbols) at different gap between the plates. In addition also the apparent stress determined with the CP geometry from Fig. 1 is given as a reference (closed symbols. The dotted line represents the theoretically calculated apparent stress in a CP geometry from the total torque of eq. (2).

The experimentally observed absolute stresses of the FMR measurements are shown in Fig. 2 as a function of the apparent shear rate $\dot{\gamma}_{n, F M R}=v / h$ for different gaps $h$ between the stationary lower plate and the upper plate that moves with velocity $v$. For comparison, Fig. 2 shows also the apparent stresses determined with the CP geometry as a function of the nominal rate $\dot{\gamma}_{n, c p}=\Omega / \tan \alpha$. The primary yield stress plateau that is observed in this ratecontrolled experiment with the $\mathrm{CP}$ geometry is $\sigma_{y} \sim 140 \mathrm{~Pa}$, and it is obvious that all stresses accessible with the FMR in Fig. 2 are still below this critical yield stress level.

The apparent flow for stresses below $\sigma_{y}$, observed both in the CP geometry as well as in the FMR, can be attributed to a slip phenomenon. It is possible to determine the slip velocities and gaps from the sliding plate data of the FMR following the general analysis of Mooney (Yoshimura and Prudhomme 1988; Cohen and Metzner 1985; Mooney 1931). The nominal 
shear rate $\dot{\gamma}_{n}$ in the gap $h$ between the shearing surfaces at a constant stress level $\sigma$ can be divided into the shear rate in the bulk phase $\dot{\gamma}_{b}$ and an apparent shear rate $\dot{\gamma}_{b}$ in the two (wall) slip layers of thickness $2 \delta$ as

$$
\dot{\gamma}_{n}=\dot{\gamma}_{b}+\frac{2 \delta}{h} \dot{\gamma}_{w}, \quad \delta<<h
$$

Linear regression of plots of the apparent shear rate $\dot{\gamma}$ as a function of the reciprocal gap $1 / h$ at constant values of the shear stress then enables the determination of $\dot{\gamma}_{b}$ as well as the slip velocities $2 v_{s}(\sigma)=2 \delta \dot{\gamma}_{w}$ at this specific shear stress level. Examples of such fits to the stress data of the FMR of Fig. 2 are shown in the appendix in Fig. 5 for different stress levels from 8 - $100 \mathrm{~Pa}$, and the obtained slip velocities as a function of the stress are given in Fig 3. It is obvious from Fig. 3 that the slip velocity is not simply showing a linear dependence on the stress as proposed by Navier (Piau and Piau 2005; Navier 1823). Instead several distinct scaling regimes can be observed with increasing stress. The onset of slip at a stress level of $\sigma_{\text {low }} \sim \sim 8 \mathrm{~Pa}$ goes along with a sudden increase of the slip velocity, which is also observed in the sudden increase in the shear rate in Fig. 2 over a relatively small stress window in particular for the smaller gap distances. Above a stress level of $10 \mathrm{~Pa}$ one observes then a power law scaling of $v_{s}=\beta \sigma^{m}$ with $m=4$ which is changing to $m=2.25$ above $\sigma \sim 20 \mathrm{~Pa}$. This scaling, that is close to the experimentally observed scaling of $v_{s} \sim \sigma^{2}$ reported for microstructured yield stress fluids as microgels (Davies and Stokes 2008) and soft sphere glasses and emulsions (Meeker et al. 2004a), extends only over a small stress window, and the slope of the $v_{s}$ vs. $\sigma$ curve continuously increases above $\sigma \sim 50 \mathrm{~Pa}$ when approaching the yield stress level $\sigma_{y}$. 


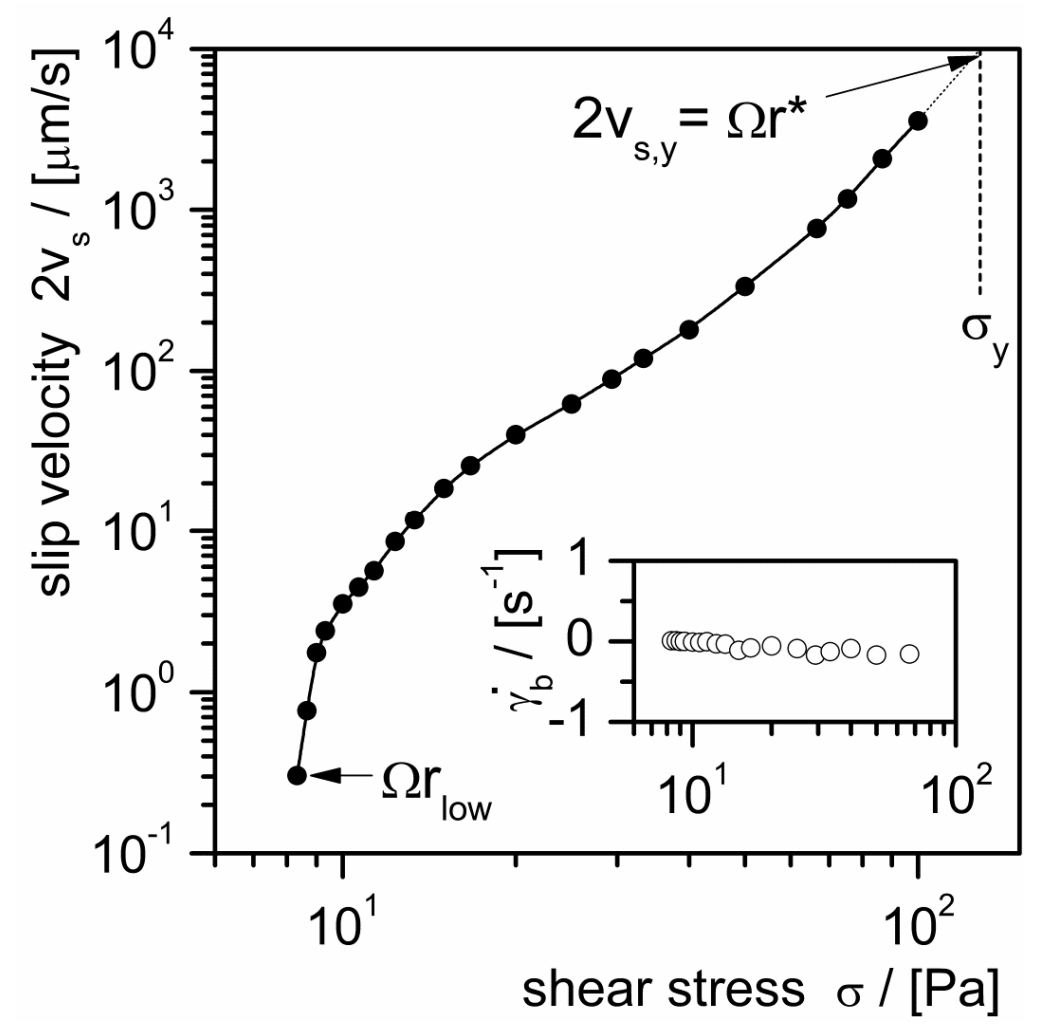

Fig. 3 Absolute slip velocities $2 v_{s}$ as function of the measured stress in the FMR. The inset shows the respective bulk shear rates $\dot{\gamma}_{b}$ as a function of the stress. The indicated critical slip velocities $\Omega r^{*}$ and $\Omega r_{l o w}$ represent the limits for the integration of eq. (2).

The bulk shear rate $\dot{\gamma}_{b}$ of the fluid is obtained from the y-axis intercept of the fits in Fig. 5. The exact values of these intercepts and thus the bulk shear rate $\dot{\gamma}_{b}$ are shown as an insert in Fig. 3 and it can clearly be seen that $\dot{\gamma}_{b}$ remains at zero for stresses below $\sigma_{y}$. Therefore, as expected for a yield stress fluid the bulk of the material remains unyielded for a stress below $\sigma_{y} \sim 140 \mathrm{~Pa}$

We are not going to make any assumption about the mechanism of the slip phenomenon in this study and the reason for the observed non-homogeneous evolution of the slip velocity in Fig. 3. Different from suspensions, emulsions or dilute polymer solutions, where the slip phenomenon can be attributed to depletion layers (Barnes 1995) of thickness $\delta$ at the walls 
and a slip layer viscosity determined by the solvent, it is not obvious if such a mechanism is also applicable for the yield stress fluid under consideration. For a concentrated polymer solution also adhesive failure with the wall (Migler et al. 1993) or a cohesive failure of the polymer at the wall and the bulk (Reimers and Dealy 1998) are possibilities discussed. The apparently sigmoidal shape of the slip velocity curve in Fig. 3 is for example similar to that expected for an adhesive failure mechanism based on a modified Eyring theory as introduced by Hatzikiriakos (Hatzikiriakos 1993). Following this model the slip velocity is $v_{s} \sim \sinh \left[\left(\sigma_{\text {low }} / \sigma-1\right) E / R T\right]$ and for a minimum amount of energy to be overcome at the interface of $\mathrm{E} \sim 1.4 \mathrm{~kJ} / \mathrm{mol}$ this model predicts the shape of the data and the apparent scaling $v_{s} \sim \sigma^{2}$ in Fig. 3 surprisingly well.

However, for the following discussion of the slipping behaviour of this fluid in a CP or PP geometry of a regular rheometer, it is not necessary to know the mechanism causing the evolution of the slip gap with stress or the viscosity in the slip gap. In order to calculate the torque $T$ measured in these geometries, it is sufficient to know the evolution of the slip velocity with stress as determined in Fig. 3. The only assumption that we make is that the slip mechanism is similar for the CP and PP geometry and the FMR, and thus depending mainly on the properties of the fluid. This assumption seems to be justified as the following comparison is indicating.

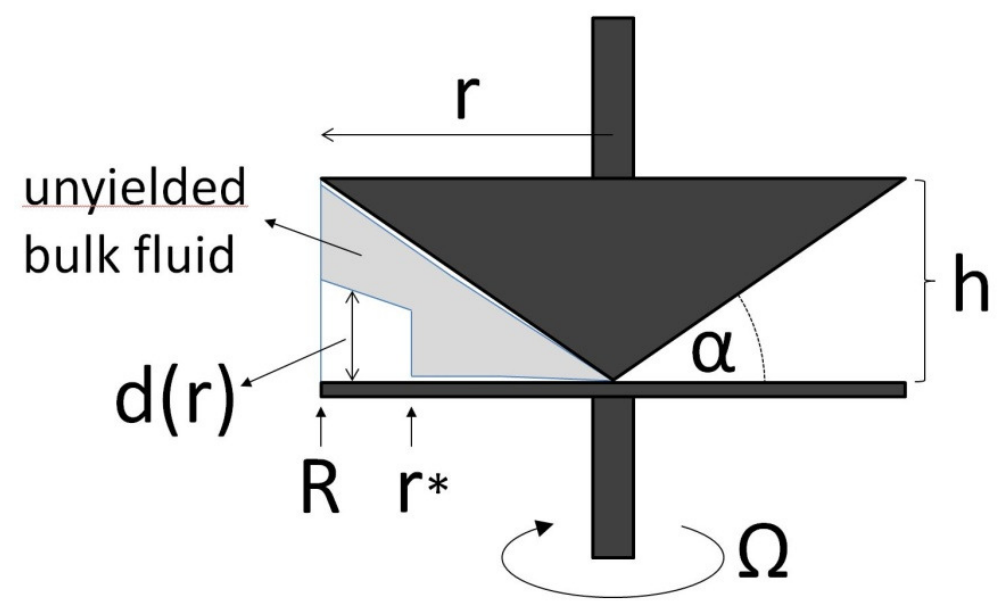


Fig. 4 Schematic drawing of the CP geometry and the partial yielding of the yield stress fluid due to a slip induced inhomogeneous radial stress distribution.

With a bulk shear rate $\dot{\gamma}_{b}=0$ it follows from eq. (1) that for such an unyielded sample the slip velocity in a rotational geometry is $2 v_{s}=\Omega r$ and therefore linearly depending on the radius. With this it follows directly from Fig. 3 that for the investigated solution the stress does not show a linear dependence on the radius as for example assumed by Russel and Grant (Russel and Grant 2000), a square root relation as proposed by Meeker et al. (Meeker et al. 2004a; Salmon et al. 2003), or a square relation as theoretically assumed amongst others by Yeow et al. (Yeow et al. 2006). For the slipping yield stress fluid in this study the stress is nonhomogeneously distributed along the radius of a CP geometry. Still, attempts to calculate the full stress distribution in a CP (or PP) geometry have used only such simplified linear or quadratic relations for the slip as mentioned above (Meeker et al. 2004a; Yeow et al. 2008) and focused more on the influence of the bulk fluid rheology (Yeow et al. 2006). In the following we reverse this approach by using the most simple bulk fluid rheology (an unyielded bulk with $\left.\dot{\gamma}_{b} \sim 0\right)$ and the true relation of stress and slip velocity $\sigma\left(2 v_{s}\right)$ or $\sigma(\Omega r)$ obtained with the sliding plate rheometer in Fig. 3. For a simple model to describe the total torque $T$ in a CP geometry as function of the angular velocity $\Omega$ of the cone it can be assumed that

$$
T(\Omega)=\int_{r^{*}}^{R} 2 \pi r^{2} \sigma_{y} d r+\int_{0}^{r^{*}} 2 \pi r^{2}[\sigma(\Omega r)] d r, \quad r^{*}<R .
$$

Here $r *$ is the critical radius above which the sample starts to yield (see also the schematic in Fig. 4), and $r^{*}$ is obtained from the slip velocity vs. stress curve of Fig. 3. As indicated in the figure the extrapolated value of the slip velocity at the yield stress is given by a critical value of $2 v_{s, y}=\Omega r^{*}$ and the critical radius $r^{*}$ is thus a function of the angular velocity $\Omega$. For low 
angular velocities for which $r^{*}=\left(2 v_{s, y} / \Omega\right) \geq R$ we assume the bulk of the material to remain fully unyielded so that eq. (2) reduces to

$$
T(\Omega)=\int_{0}^{R} 2 \pi r^{2}[\sigma(\Omega r)] d r, \quad r^{*} \geq R
$$

On the other hand, for $r^{*}<r<R$ the fluid will start to partially yield in the outer regimes of the CP geometry and form a banded structure, as schematically indicated in Fig. 4. The stress remains in this banded regime constant at $\sigma_{y}$, independent of the angular velocity or radial position. The only thing that changes is the width $d(r)$ of the yielded band (also indicated in Fig. 4) in which the liquid flows (or the thickness $h-d$ of the unyielded band) (Picard et al. 2002). The shear rate in this gap will also stay constant at $\dot{\gamma}_{y}=\Omega r / d$, determined by the viscosity of the yielded sample at this yield stress $\eta\left(\sigma_{y}\right)$. For a given angular velocity the ratio $r / d$ is therefore constant along the radial axis, and decreases with increasing $\Omega$. The upper limit of the yielding regime in this simplified model is reached at a critical angular velocity of $\Omega_{c}=\dot{\gamma}_{y} \tan \alpha$ where the ratio $r / d$ has reached the limit $r / h=\tan \alpha$ and the fluid is fully yielded and the unyielded band has disappeared. Beyond this critical angular velocity the total torque is determined by the general relation for a CP geometry $T(\Omega)=\sigma(\Omega \tan \alpha) 2 \pi R^{3} / 3$ and thus solely depending on the stress function of the fully yielded liquid $\sigma(\Omega \tan \alpha)$ or $\sigma\left(\dot{\gamma}_{n, c p}\right)$.

For a comparison of this model to experimental data the total torque $T$ as a function of the angular velocity is calculated from eqs. (2) and (3), using the experimental results of Fig. 3 for the stress function $[\sigma(\Omega r)]$ in the second term of eqs. (2) and integrating numerically over the range $r_{\text {low }}$ to $r^{*}$ that is indicated in Fig. 3. This result for the torque is converted to the 
apparent (constant) stress in a non-slipping CP geometry, $\sigma=3 T / 2 \pi R^{3}$, and compared in Fig. 2 to the measured apparent stress in the $\mathrm{CP}$ geometry as a function of the nominal shear rate $\dot{\gamma}_{n, c p}=\Omega / \tan \alpha$. The comparison to the experimentally obtained data for the CP geometry shows that this simple model gives a good agreement over the range of apparent shear rates up to the yield stress level of the fluid. The deviation at rates below $0.15 \mathrm{~s}^{-1}$ can be attributed to the fact that the lower limit for the numerical integration is not 0 but the experimental limit $r_{\text {low }}$ of the FMR measurements. The contribution of stresses from fluid elements at small CP radii with slip velocities $2 v_{s}<\Omega r *$ is thus not included in the theoretically predicted stresses, and the model is therefore underpredicting the stress at low rates as can be seen in Fig. 3. A calculation of the evolution of stresses above the yield stress level and $\Omega_{c}$ is not included, as the necessary stress function $\sigma(\Omega \tan \alpha)$ would be obtained from the experimental CP data to which the prediction would be compared to. This would obviously result in a perfect match, and the numerical calculations are thus limited solely to stresses obtained from the slip velocities of the FMR, resulting in the observed plateau at $\sigma_{y}$ also at higher rates.

From the definitions of the nominal shear rates used for the cone-and-plate geometry, $\dot{\gamma}_{n, c p}=\Omega / \tan \alpha$, and for the plate-and-plate geometry, $\dot{\gamma}_{n, p p}=\Omega R / h$, it follows that the same apparent shear rate at the same angular velocity in the CP and PP geometry will be observed for a gap distance in the PP geometry of $h=R \tan \alpha$. For the given geometry radius and angle of the geometries in this study this gap calculates to $h=698 \mu \mathrm{m}$, and in Fig. 1 it is obvious that in the pre-yield slip regime the curves for the CP and the PP geometry at a gap of $h=500$ $\mu \mathrm{m}$ are nearly coinciding. Furthermore, it can be seen in Fig. 1 that the pre-yield curves for PP geometry measurements at lower gaps $h$ have the same shape and are just shifted along the rate axis by $\dot{\gamma}_{n, p p}=(h / R \tan \alpha) \dot{\gamma}_{n, c p}$. 
A close observation of the PP data at stresses above $\sigma_{y}$ shows that also here an apparent shift of the lower gap data along the x-axis is observed. This can also be attributed to a slip of the fluid, however, now of the yielded sample. This is likely to follow a different mechanism, as the shift is much smaller than observed for the stresses below $\sigma_{y}$. A precise analysis of this high-rate slip with the FMR, as done for the low rates, is not possible as these stresses exceed the technical limits of the thin film rheometer. On the other hand it demonstrates the capability of the FMR to investigate in particular slip velocities at low nominal rates and stresses as observed and expected in polymer solutions, suspensions and other complex fluids with fragile microstructures.

\section{Conclusions}

In this paper we have demonstrated the capabilities of the sliding plate configuration of the FMR rheometer to determine the absolute relation between the slip velocity and the stress for a yield stress fluid. In particular the sensitive compound flexures of the FMR in combination with the possibility to achieve precise gap settings down to $1 \mu \mathrm{m}$ allows to accurately determining slip velocities down to $1 \mu \mathrm{m} / \mathrm{s}$. The FMR is therefore ideally suited to investigated slip phenomena with its sliding plate configuration for complex fluids as suspensions, emulsions or solutions of lower viscosities and yield stresses, in the current configuration down to stresses of $2 \mathrm{~Pa}$ (that were so far not accessible with the sliding plate rheometer of Dealy and co-workers developed for polymer melts).

The FMR has been used to precisely determine the non-linear relation of the slip velocity to stress for a concentrated xanthan gum solution as a model yield stress material. This relation allowed calculating the non-homogeneous radial stress distribution of the slipping xanthan gum solution in a cone-and-plate geometry. With this and the assumption of a partial yielding of the slipping sample it was possible to predict the apparent stress or torque in the CP 
geometry as function of the applied rates up to the yield stress level. A comparison of these calculations to measured apparent stresses with the CP geometry showed good agreement, indicating the validity of the used simple model assumptions.

\section{Acknowledgements}

The authors would like to acknowledge financial support from the research foundation

Flanders (FWO) (project G.0543.10). They also acknowledge support by the Bijzonder

Onderzoeksfonds K.U. Leuven (GOA 09/002) Furthermore they would like to thank G.H.

McKinley and P. Erni for fruitful discussions.

\section{References}

Ardakani HA, Mitsoulis E, Hatzikiriakos SG (2011) Thixotropic flow of toothpaste through extrusion dies. J Non-Newton Fluid Mech 166 (21-22):1262-1271. doi:10.1016/j.jnnfm.2011.08.004

Baik SJ, Moldenaers P, Clasen C (2008) Determination of normal stresses in micrometer thin films. In: Co A, Leal LG, Colby RH, Giacomin AJ (eds) Xvth International Congress on Rheology - the Society of Rheology 80th Annual Meeting, Pts 1 and 2, vol 1027. Aip Conference Proceedings. Amer Inst Physics, Melville, pp 1165-1167

Baik SJ, Moldenaers P, Clasen C (2011) A sliding plate microgap rheometer for the simultaneous measurement of shear stress and first normal stress difference. Rev Sci Instrum 82 (3):035121. doi:10.1063/1.3571297

Barnes HA (1995) A Review of the Slip (Wall Depletion) of Polymer-Solutions, Emulsions and Particle Suspensions in Viscometers - Its Cause, Character, and Cure. J Non-Newton Fluid Mech 56 (3):221-251

Bertola V, Bertrand F, Tabuteau H, Bonn D, Coussot P (2003) Wall slip and yielding in pasty materials. J Rheol 47 (5):1211-1226. doi:10.1122/1.1595098

Clasen C (2012) High shear rheometry using hydrodynamic lubrication flows. J Rheol submitted

Clasen C, Gearing BP, McKinley GH (2006) The flexure-based microgap rheometer (FMR). J Rheol 50 (6):883-905

Clasen C, Kavehpour HP, McKinley GH (2010) Bridging Tribology And Microrheology Of Thin Films. Appl Rheol 20 (4):196-208. doi:10.3933/ApplRheol-20-45049

Clasen C, McKinley GH Microrheometry: Gap-Dependent Rheology and Tribology of Complex Fluids. In: Lee JW, Lee SJ (eds) The XIVth International Congress on Rheology, Seoul, 2004. The Korean Society of Rheology, pp 1-3

Cohen Y, Metzner AB (1985) Apparent Slip-Flow of Polymer-Solutions. J Rheol 29 (1):67-102

Davies GA, Stokes JR (2008) Thin film and high shear rheology of multiphase complex fluids. J NonNewton Fluid Mech 148 (1-3):73-87

Derakhshandeh B, Hatzikiriakos SG, Bennington CPJ Rheology of pulp suspensions using ultrasonic Doppler velocimetry. Rheol Acta 49 (11-12):1127-1140. doi:10.1007/s00397-010-0485-2

Dimitriou CJ, McKinley GH, Venkatesan R (2011) Rheo-PIV Analysis of the Yielding and Flow of Model Waxy Crude Oils. Energy Fuels 25 (7):3040-3052. doi:10.1021/ef2002348 
Erni P, Varagnat M, Clasen C, Crest J, McKinley GH (2011) Microrheometry of sub-nanolitre biopolymer samples: non-Newtonian flow phenomena of carnivorous plant mucilage. Soft Matter 7 (22):10889-10898. doi:10.1039/c1sm05815k

Giacomin AJ, Samurkas T, Dealy JM (1989) A Novel Sliding Plate Rheometer for Molten Plastics. Polym Eng Sci 29 (8):499-504

Hatzikiriakos SG (1993) A slip model for linear-polymers based on adhesive failure. Int Polym Process $8(2): 135-142$

Hatzikiriakos SG, Dealy JM (1991) Wall Slip of Molten High-Density Polyethylene .1. Sliding Plate Rheometer Studies. J Rheol 35 (4):497-523

Kalika DS, Denn MM (1987) Wall slip and extrudate distortion in linear low-density polyethylene. J Rheol 31 (8):815-834. doi:10.1122/1.549942

Kalyon DM, Yaras P, Aral B, Yilmazer U (1993) Rheological behaviour of a concentrated suspension - A solid rocket fuel simulant. J Rheol 37 (1):35-53. doi:10.1122/1.550435

Kojic N, Bico J, Clasen C, McKinley GH (2006) Ex vivo rheology of spider silk. Journal of Experimental Biology 209 (21):4355-4362

Kramer J, Uhl JT, Prudhomme RK (1987) Measurement of the Viscosity of Guar Gum Solutions to 50,000 1/s Using a Parallel Plate Rheometer. Polym Eng Sci 27 (8):598-602

Laun HM (2004) Capillary rheometry for polymer melts revisited. Rheol Acta 43 (5):509-528. doi:10.1007/s00397-004-0387-2

Lumma D, Best A, Gansen A, Feuillebois F, Radler JO, Vinogradova OI (2003) Flow profile near a wall measured by double-focus fluorescence cross-correlation. Phys Rev E 67 (5):10. doi:056313

10.1103/PhysRevE.67.056313

Macosko C (1994) Rheology: Principles, Measurements, and Applications. VCH Publishers, New York

Mair RW, Callaghan PT (1996) Observation of shear banding in worm-like micelles by NMR velocity imaging. Europhys Lett 36 (9):719-724. doi:10.1209/epl/i1996-00293-9

Manneville S, Becu L, Colin A (2004) High-frequency ultrasonic speckle velocimetry in sheared complex fluids. Eur Phys J-Appl Phys 28 (3):361-373. doi:10.1051/epjap:2004165

Meeker SP, Bonnecaze RT, Cloitre M (2004a) Slip and flow in pastes of soft particles: Direct observation and rheology. J Rheol 48 (6):1295-1320. doi:10.1122/1.1795171

Meeker SP, Bonnecaze RT, Cloitre M (2004b) Slip and flow in soft particle pastes. Phys Rev Lett 92 (19):4. doi:198302

10.1103/PhysRevLett.92.198302

Migler KB, Hervet H, Leger L (1993) Slip Transition of a Polymer Melt under Shear-Stress. Phys Rev Lett 70 (3):287-290

Mooney M (1931) Explicit formulas for slip and fluidity. J Rheol 2:210-222

Navier CLMH (1823) On the laws of movement of fluids. Mém de l'Acad Roy des Sciences de l'Inst de France 6:389-440

Neto C, Evans DR, Bonaccurso E, Butt HJ, Craig VSJ (2005) Boundary slip in Newtonian liquids: a review of experimental studies. Rep Prog Phys 68 (12):2859-2897. doi:10.1088/0034$4885 / 68 / 12 / \mathrm{r} 05$

Piau JM, Piau A (2005) Letter to the Editor: Comment on "Origin of concentric cylinder viscometry" [J. Rheol. 49, 807-818 (2005)]. The relevance of the early days of viscosity, slip at the wall, and stability in concentric cylinder viscometry. J Rheol 49 (6):1539-1550. doi:10.1122/1.2072087

Picard G, Ajdari A, Bocquet L, Lequeux F (2002) Simple model for heterogeneous flows of yield stress fluids. Phys Rev E 66 (5):12. doi:051501

10.1103/PhysRevE.66.051501

Princen HM, Kiss AD (1986) Rheology of foams and highly concentrated emulsions. III: Static shear modulus. J Colloid Interface Sci 112 (2):427-437. doi:10.1016/0021-9797(86)90111-6

Reimers MJ, Dealy JM (1998) Sliding plate rheometer studies of concentrated polystyrene solutions: Nonlinear viscoelasticity and wall slip of two high molecular weight polymers in tricresyl phosphate. J Rheol 42 (3):527-548 
Rofe CJ, deVargas L, PerezGonzalez J, Lambert RK, Callaghan PT (1996) Nuclear magnetic resonance imaging of apparent slip effects in xanthan solutions. J Rheol 40 (6):1115-1128. doi:10.1122/1.550775

Russel WB, Grant MC (2000) Distinguishing between dynamic yielding and wall slip in a weakly flocculated colloidal dispersion. Colloid Surf A-Physicochem Eng Asp 161 (2):271-282. doi:10.1016/s0927-7757(99)00376-3

Salmon JB, Becu L, Manneville S, Colin A (2003) Towards local rheology of emulsions under Couette flow using Dynamic Light Scattering. Eur Phys J E 10 (3):209-221. doi:10.1140/epje/i200210110-5

Seth JR, Cloitre M, Bonnecaze RT (2008) Influence of short-range forces on wall-slip in microgel pastes. J Rheol 52 (5):1241-1268. doi:10.1122/1.2963135

Yeow YL, Leong YK, Khan A (2006) Non-Newtonian flow in parallel-disk viscometers in the presence of wall slip. J Non-Newton Fluid Mech 139 (1-2):85-92. doi:10.1016/j.jnnfm.2006.07.005

Yeow YL, Leong YK, Khan A (2008) Slow steady viscous flow of newtonian fluids in parallel-disk viscometer with wall slip. J Appl Mech-Trans ASME 75 (4):7. doi:041001

10.1115/1.2910901

Yoshimura A, Prudhomme RK (1988) Wall Slip Corrections for Couette and Parallel Disk Viscometers. J Rheol 32 (1):53-67

\section{Appendix}

The slip velocity and bulk shear rate are determined following the Mooney analysis by plotting for a constant stress the different shear rates that were observed in Fig. 2 at different gaps as function of the inverse gap. Following eq. (1) the linear extrapolation of such a series of constant stress gives the bulk shear rate as the y-axis intersect and the slip velocity as the slope of the interpolated curve. 

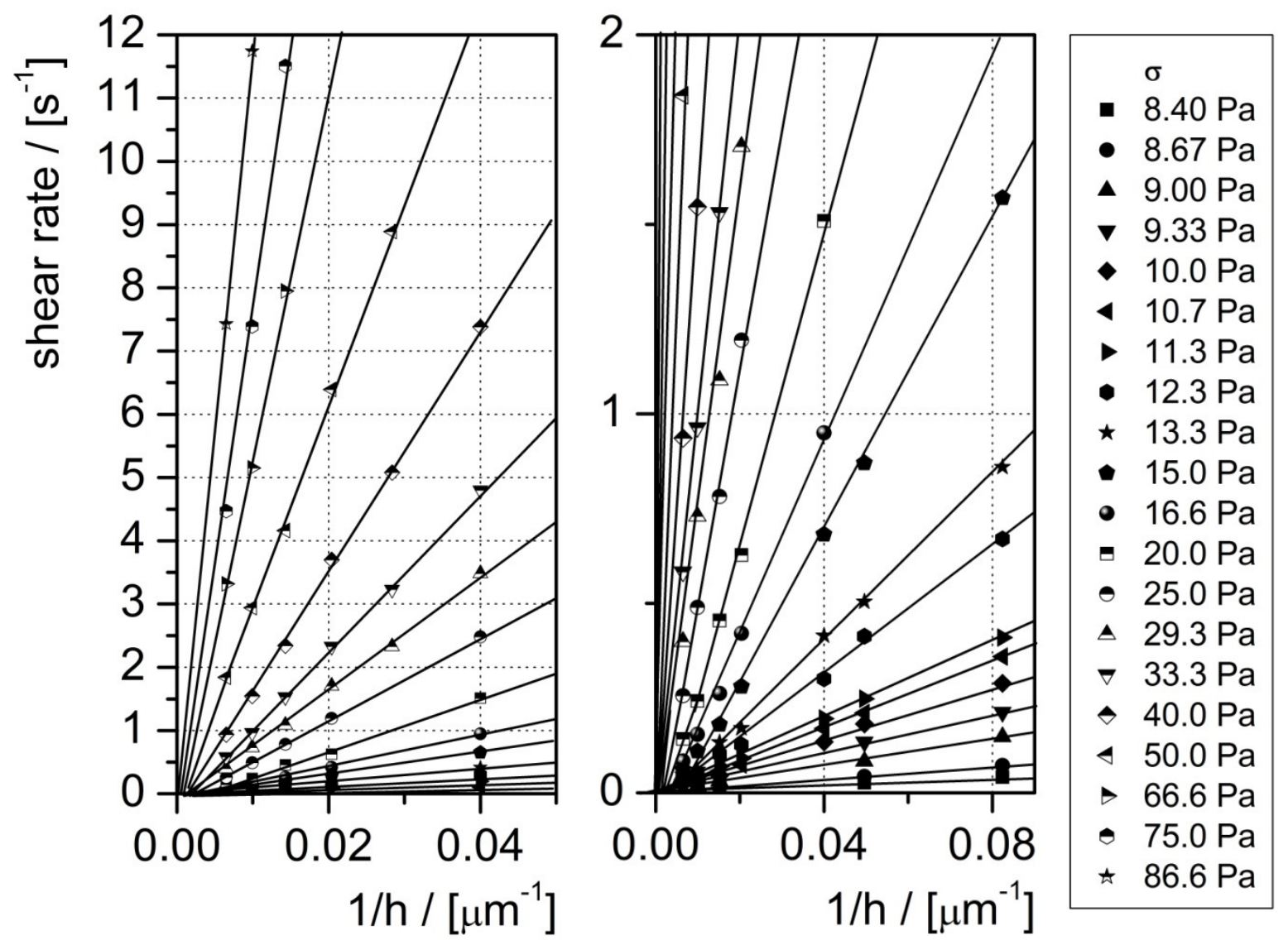

Fig. 5 Slip analysis of the flow curve data of Fig. 2 
Figures

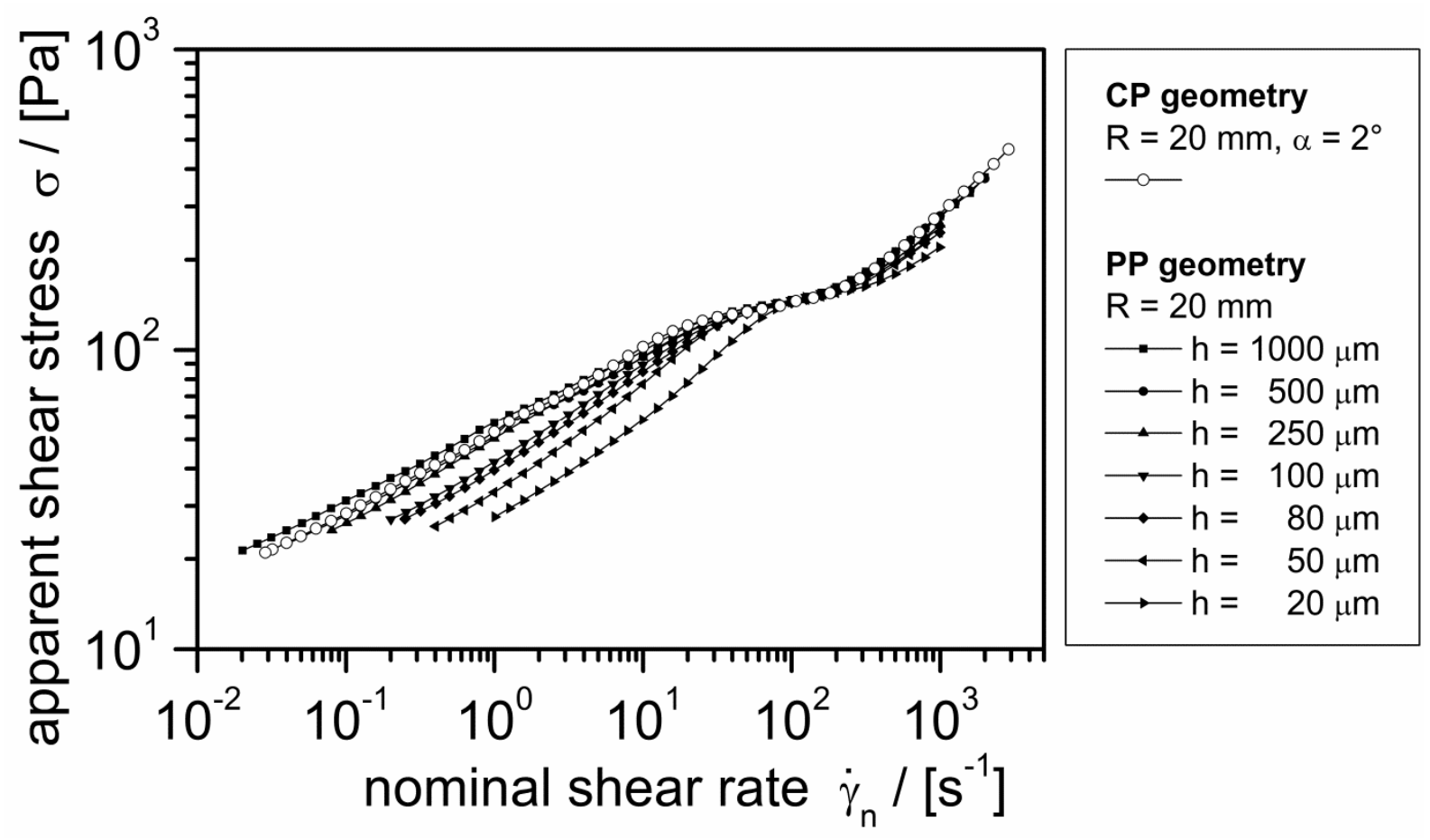

Fig. 1 Apparent shear stress of the $5 \mathrm{wt} \%$ aqueous xanthan gum solution determined with a cone-andplate (CP) geometry as well as a plat-and-plate (PP) geometry at different gaps $h$, as a function of the nominal shear rate of the respective geometry. 


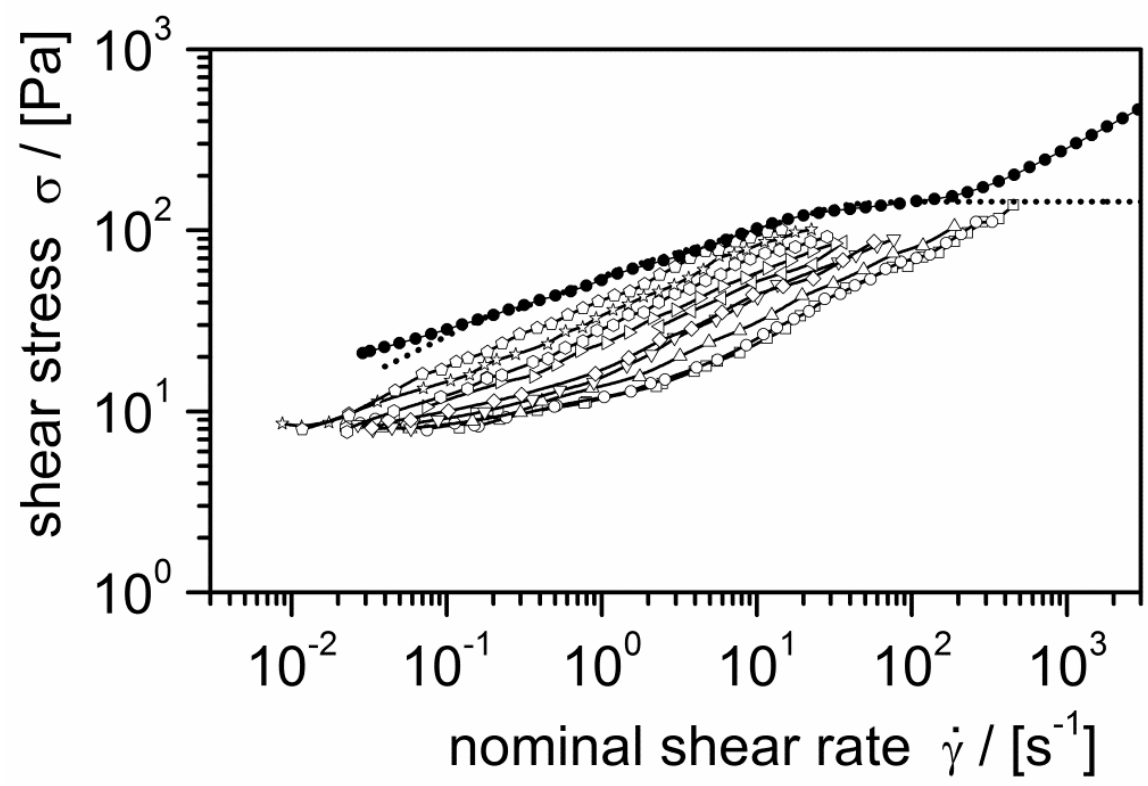

absolute shear stress from the FMR:

$\square-\mathrm{h}=4.51 \mu \mathrm{m}$

$\longrightarrow \mathrm{h}=6.92 \mu \mathrm{m}$

$\longrightarrow \mathrm{h}=12.1 \mu \mathrm{m}$

$\longrightarrow-\mathrm{h}=20.2 \mu \mathrm{m}$

$\longrightarrow \mathrm{h}=25.0 \mu \mathrm{m}$

$\neg-\mathrm{h}=35.2 \mu \mathrm{m}$

$\longrightarrow \mathrm{h}=50.1 \mu \mathrm{m}$

$-\mathrm{h}=78.1 \mu \mathrm{m}$

$\longrightarrow-\mathrm{h}=100 \mu \mathrm{m}$

$\multimap \mathrm{h}=154 \mu \mathrm{m}$

apparent shear stress:

- - - CP geometry

........ calculated

Fig. 2 Absolute shear stresses of the $5 \mathrm{wt} \%$ aqueous xanthan gum solution as a function of the nominal shear rates determined with the sliding plate geometry of the FMR (open symbols) at different gap between the plates. In addition also the apparent stress determined with the CP geometry from Fig. 1 is given as a reference (closed symbols. The dotted line represents the theoretically calculated apparent stress in a CP geometry from the total torque of eq. (2). 


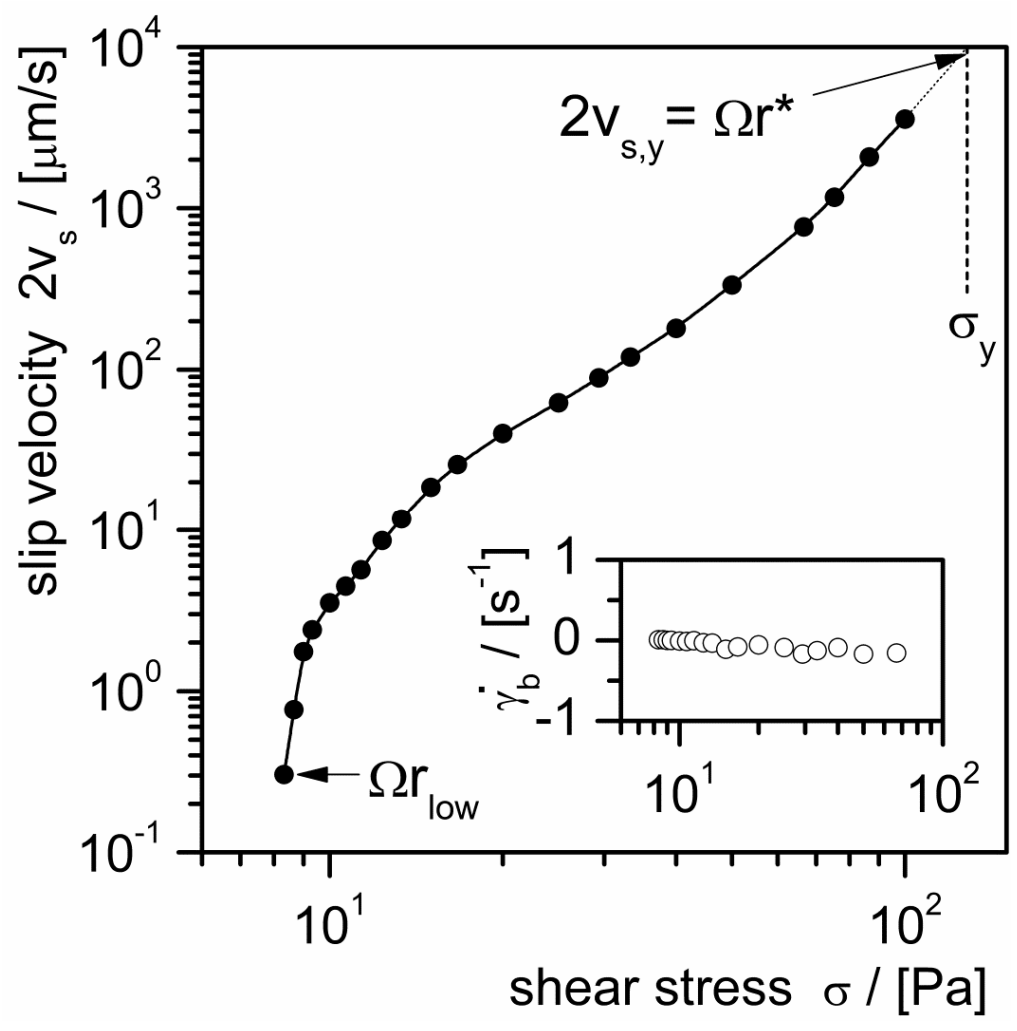

Fig. 3 Absolute slip velocities $2 v_{s}$ as function of the measured stress in the FMR. The inset shows the respective bulk shear rates $\dot{\gamma}_{b}$ as a function of the stress. The indicated critical slip velocities $\Omega r^{*}$ and $\Omega r_{l o w}$ represent the limits for the integration of eq. (2). 


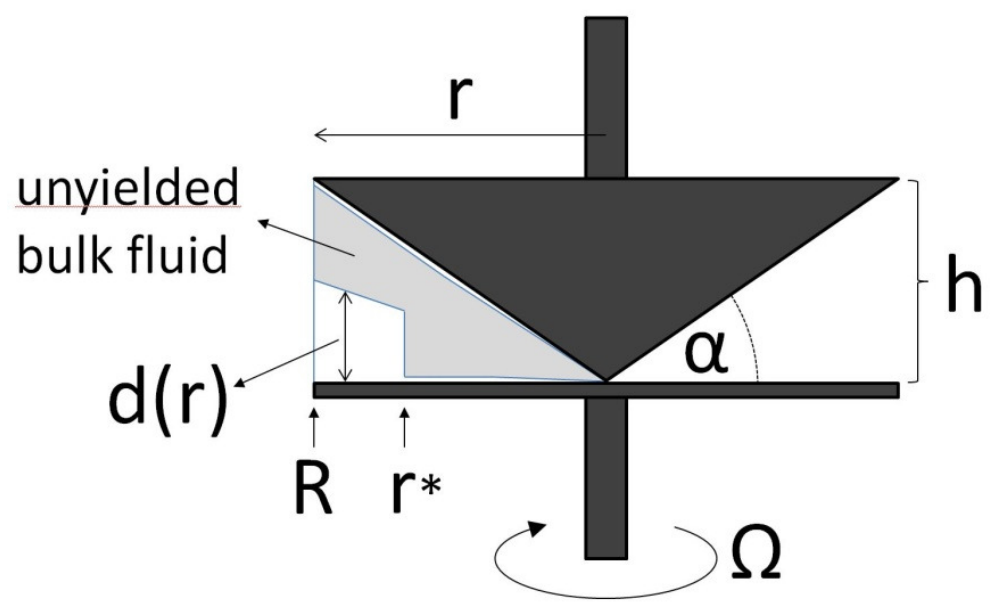

Fig. 4 Schematic drawing of the CP geometry and the partial yielding of the yield stress fluid due to a slip induced inhomogeneous radial stress distribution. 

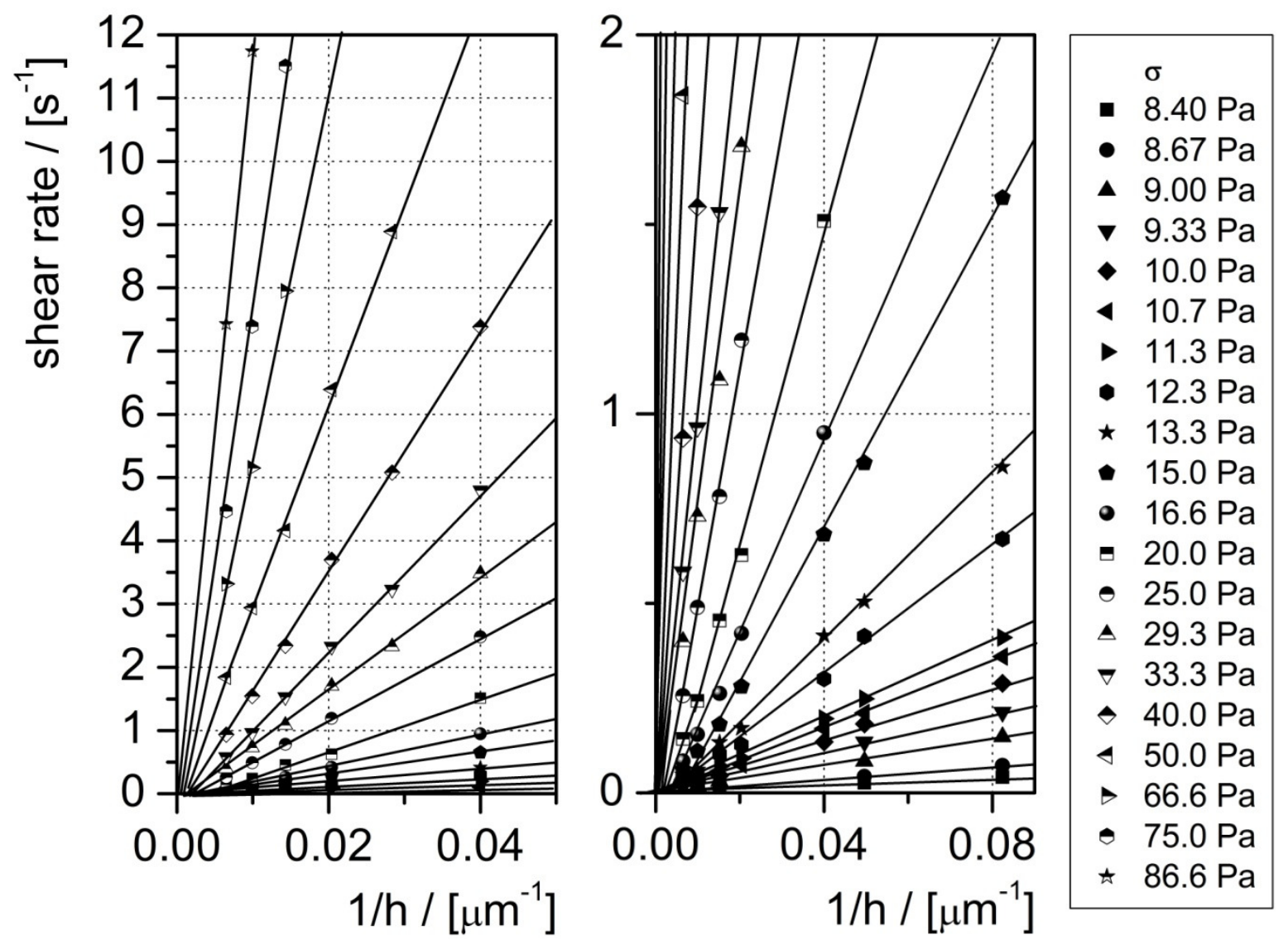

Fig. 5 Slip analysis of the flow curve data of Fig. 2 